into scale-up of the program and are building the informatics tools that will ultimately allow our microbial risk scores to be made available to clinicians via a smart-phone enabled app. The primary objective of this work is to enable the precision management of infections using isolate-specific virulence data. This PIM approach will inform clinical decision-making and infection management practices in point-of-care settings resulting in, (1) a reduction in the number of people who develop life-threatening infections, (2) a reduction in the number of side effects that result from over-treating benign infections, and (3), an extended service-life for our existing antibiotics by dramatically reducing the over-use of these drugs. We introduce a new Precision Infection Management (PIM) strategy for titrating clinical care according to the risks posed by each individual infection.

Disclosure No significant relationships.

\section{S15.4 COMBATTING HIV WITH NANOMATERIALS}

Kim A Woodrow*. University of Washington, Bioengineering, Seattle, USA

\subsection{6/sextrans-2019-sti.72}

The delivery of drug combinations is a paradigm for treatment of cancer, HIV/AIDS and drug resistant bacterial infections. My laboratory is interested in the application of engineered biomaterials to control the spatial and temporal delivery of a combination of agents (small molecules, biologics, conjugates). Strategies to combine chemically incompatible agents may facilitate the discovery of unique drug-drug activities, particularly unexplored combination drug synergy. In this presentation, I will summarize our efforts to develop polymeric delivery systems for the combination delivery of antiretroviral (ARV) drugs in HIV prevention, treatment and cure. We have developed polymeric particulate and fiber carrier systems for delivering ARV drug combinations. The flexibility to design the nanoarchitecture of these polymeric carriers, combined with the versatility of drugs that can be encapsulated for controlled release, motivate the use of these systems for topical, injectable or oral delivery of combination agents.

\section{S16.1 - ANATOMICAL SITES OF INFECTION: BIOMEDICAL, MODELING, BEHAVIORAL, AND PROGRAMMATIC CONSIDERATIONS FOR STI PREVENTION}

\section{Wednesday, July 17, 2019 10:45 AM - 12:15 PM}

\section{S16.1 ANATOMICAL SITES OF INFECTION - BIOMEDICAL CONSIDERATIONS FOR STI PREVENTION}

Jane Hocking*. University of Melbourne, Melbourne School of Population and Global Health, Carlton, Australia

10.1136/sextrans-2019-sti.73

Extragenital (anorectal and oropharyngeal) sexually transmitted infections (STIs), particularly chlamydia and gonorrhoea, are highly prevalent among men who have sex with men (MSM), but are now an increasing concern among heterosexual men and women with calls for anorectal testing in women. Further, there is ongoing debate about the role of oropharyngeal STIs driving transmission, particularly among MSM and about anorectal chlamydia in women causing urogenital infection via auto-inoculation. These issues highlight that site of infection is an important issue. Treatment efficacy can vary considerably by site of infection - for example, treatment efficacy is lower for oropharyngeal gonorrhoea and anorectal chlamydia with some treatments. Factors related to the pharmacokinetic properties of the drug can affect its efficacy at different anatomic sites including its tissue distribution, protein binding and half-life. Factors related to the individual including the $\mathrm{pH}$ of the local tissue environment, immune response, drug side-effects and sexual practices can affect treatment efficacy. Finally, factors related to the microorganism itself such as organism load and antimicrobial resistance can also impact on treatment efficacy. These factors should play a role in guiding treatment guidelines as it is possible that treatment regimens need to vary by site of infection. The use of mouthwash and doxycycline prophylaxis have been raised as potential biomedical interventions to reduce STI transmission, although ongoing concern about antimicrobial stewardship threatens the widespread use of doxycycline. We also need to understand the natural history of extra-genital STIs, particularly in women where the importance of anorectal infections and whether they play an important role in inoculating and causing persistent urogenital infection is not well understood. This presentation will discuss the importance of the site of infection particularly when considering treatment options and the possible role of biomedical interventions to prevent infection.

Disclosure No significant relationships.

\section{S16.2 MODELING CONSIDERATIONS RELATED TO MULTI-SITE INFECTION}

Ian Spicknall*. Centers for Disease Control and Prevention, Atlanta, USA

\subsection{6/sextrans-2019-sti.74}

Background Gonorrhea may infect the urethra, rectum, and oropharynx in men. It may only be acquired when there is contact between infected and uninfected anatomical sites. Seven plausible routes of MSM transmission have been proposed: urethra-to-rectum, rectum-to-urethra, urethra-to-oropharynx, rectum-to-oropharynx, oropharynx-to-urethra, oropharynx-to-rectum, and oropharynx-to-oropharynx. We characterize the uncertainty and potential importance of transmission from each anatomical site using a deterministic compartmental mathematical model.

Methods We developed a model of site-specific gonococcal infection, where individuals are infected at zero, one, two, or all three sites. Sexual behavior and infection duration parameters were fixed similar to recent analyses. Markov Chain Monte Carlo methods were used to sample the posterior distribution of transmission probabilities that were consistent with site-specific prevalence in American MSM populations under specific scenarios. Scenarios were defined by whether transmission routes may or may not transmit by constraining specific transmission probabilities to zero rather than fitting them. 
Results Transmission contributions from each site have greater uncertainty when more routes may transmit, when all routes may transmit, the oropharynx can contribute $0-100 \%$ of all transmissions. In contrast, when only anal or oral sex may transmit, transmission from the oropharynx can account for only $0-25 \%$ of transmission. Intervention effectiveness against transmission from each site also has greater uncertainty when more routes may transmit.

Conclusion Multiple routes of transmission leads to great uncertainty. Even under ideal conditions (i.e., when site-specific gonococcal prevalence, relative rates of specific sex acts, and duration of infection at each anatomical site are known and do not vary), the relative importance of different anatomical sites for gonococcal transmission cannot be inferred with precision. This result is generalizable to any other infection where multi-site infection leads to multiple routes of transmission. Additional data informing per act transmissibility are needed to understand site-specific gonococcal infection transmission. This understanding is essential for predicting population-specific intervention effectiveness.

Disclosure No significant relationships.

\section{S16.3 ANATOMICAL SITES OF INFECTION: BEHAVIOURAL CONSIDERATIONS FOR STI PREVENTION}

Catherine Mercer*. University College London, Centre for Population Research in Sexual Health and HIV, Institute for Global Health, London, UK

\subsection{6/sextrans-2019-sti.75}

Many industrialised countries have witnessed a broadening of sexual repertoires, including increases in reported heterosexual oral, and in particular, anal sex, and same-sex behaviour in women, while among MSM, oral sex remains more prevalent than anal sex. Condoms, when used correctly, are highly-effective in preventing STI transmission through penetrative sex, yet their use remains suboptimal. For MSM, this may partly reflect the effectiveness of biomedical interventions for HIV such as treatment as prevention and pre-exposure prophylaxis. For heterosexuals, pregnancy prevention often trumps STI concerns, with more reliable and less intrusive contraception used for vaginal sex, while condoms are seldom used for heterosexual anal sex, or oral sex regardless of gender.

Given these behavioural trends, it is unsurprising that a large proportion of STI transmission is thought to occur extra-genitally. Among MSM attending US sexual health clinics, more than half of GC/CT infections were not in the urethra, and most MSM with extra-genital GC/CT infections did not have concurrent urethral infections. Extra-genital infections are more often asymptomatic, a potential reservoir for transmission, and undetected antibiotic resistant strains may spread resistance. STI prevention efforts must therefore include targeting extra-genital infections.

Efforts to change sexual practice, e.g. promoting condom use for oral sex and/or sexual positioning, are unlikely to have significant impacts, but opportunities exist beyond the bedroom. Raising public awareness about the potential for, and consequences of, extra-genital infection may encourage disclosing sexual behaviour to clinicians and appropriate sitespecific testing. Educating clinicians - especially non-specialists - about the importance of asking all patients about their sexual practices and testing for extra-genital STIs accordingly may also be helpful. Such endeavours could result in the greater detection of extra-genital infections but cost-effective strategies need determining. As such, a multifaceted approach including evidence-based behavioural and biomedical interventions is likely to yield the greatest health gains.

Disclosure No significant relationships.

\section{S16.4 DESIGNING AN APPROACH TO DEAL WITH EXTRAGENITAL SEXUALLY TRANSMITTED INFECTIONS: DO WE HAVE THE DATA WE NEED?}

Jeanne Marrazzo*. University of Alabama, Medicine, Birmingham, USA

\subsection{6/sextrans-2019-sti.76}

This symposium entitled 'Anatomical Sites of Infection: Biomedical, Modeling, Behavioral, and Programmatic Considerations for STI Prevention' will focus on the implications that extragenital (defined as infections that occur outside of the cervix and urethra) sexually transmitted infections have for various dimensions of STI care and prevention. These infections are common, particularly among men who have sex with men (rectal and pharyngeal) and heterosexual women (both sites as well). Yet, we have little understanding of the pharmacokinetics of commonly used antimicrobials at these sites, the natural history of the infections at these sites, and as a critical corollary, how much of a role these sites have in providing a meaningful reservoir for sustaining transmission in populations at risk. We need to have a better understanding of these parameters before we can intelligently design screening protocols and intervention studies. This presentation will explore these issues and allow ample time for discussion of these challenges.

Disclosure No significant relationships.

\section{S17 - CLINICAL ISSUES IN WOMEN'S HEALTH AND STI}

Wednesday, July 17, 2019
10:45 AM - 12:15 PM

\section{S17.1 HPV SCREENING - NEW EVIDENCE AND CURRENT STATE OF THE ART}

Suzanne Garland*. The University of Melbourne, Obstetrics and Gynaecology, Parkville, Australia

\subsection{6/sextrans-2019-sti.77}

HPV-vaccination programs constitute major public-health initiatives worldwide and have been introduced into National immunisation programs in over 80 countries, although most are in high income countries. Programs were implemented around 10 years ago: where high coverage of target populations especially with catch-up programs, the impact and effectiveness on HPV infection and disease has been remarkable. For the quadrivalent vaccine $(6 / 11 / 16 / 18)$ there have been reductions of $\sim 90 \%$ for $\mathrm{HPV}$ vaccine type infections, $\sim 90 \%$ for genital warts, $\sim 45 \%$ for low-grade cytological cervical abnormalities, $\sim 60 \%$ for cervical histologically-proven highgrade abnormalities [HSIL], in colposcopic referrals, and 